\title{
Multiple lectin assays in detecting glycol-alteration status of serum NRG1 in papillary thyroid cancer
}

\author{
Wan-Lin Liu ${ }^{1}$, Yi-Ming Cao ${ }^{1}$, Tian Liao ${ }^{2}$, Ning Qu ${ }^{1}$, Yong-Xue Zhu ${ }^{1}$, Wen-Jun Wei ${ }^{1}$ \\ ${ }^{1}$ Department of Head and Neck Surgery, Fudan University Shanghai Cancer Center, Shanghai, China; ${ }^{2}$ Department of Oncology, Shanghai Medical \\ College, Fudan University, Shanghai, China \\ Contributions: (I) Conception and design: YX Zhu, WJ Wei; (II) Administrative support: None; (III) Provision of study materials or patients: WL Liu, \\ T Liao, N Qu; (IV) Collection and assembly of data: WL Liu, YM Cao; (V) Data analysis and interpretation: WJ Wei; (VI) Manuscript writing: All \\ authors; (VII) Final approval of manuscript: All authors. \\ Correspondence to: Yong-Xue Zhu. Department of Head and Neck Surgery, Fudan University Shanghai Cancer Center, No. 270, Dong’An Road, \\ Shanghai, China. Email: wonisbesing@hotmail.com; Wen-Jun Wei. Department of Head and Neck Surgery, Fudan University Shanghai Cancer \\ Center, No. 270, Dong'An Road, Shanghai, China. Email: kakarwen@163.com.
}

\begin{abstract}
Background: Neuregulin 1 (NRG1) is a membrane glycoprotein mediating cell-to-cell signaling and has a crucial role in the growth and development of various organ systems. Our study explored its diagnostic value in distinguishing BRAF V600E mutant status in papillary thyroid cancer (PTC) patients by analyzing multiple glycan patterns of serum NRG1 through lectin assays.

Methods: We first extracted serum from PTC patients and tested BRAF V600E mutation by immunohistochemical (IHC) staining. Then we applied antibody overlay lectin microarray and lectin blot to detect glycol-alterations of NRG1. Then Aleuria aurantia lectin (AAL) ELISA was performed according to ELISA index to test the protein fucosylation level of NRG1 (Fuc-NRG1).

Results: We got glycan profiles of 14 lectins, including GNL, GSL2, AAL, BPL, ECL, CAL, NML, HHL, PHA-L, RCA-I, ConA, DBA, PWA and LEL. Six of them, namely, GSL2, BPL, NML, HHL, PHA-L and LEL, had significantly increased binding affinity capacity in BRAF(+) PTC compared with BRAF(-) PTC controls. LEL, BPL and NML tended to bind to NRG1 in BRAF(+) PTC group. Both AAL ELISA and protein ELISA assays showed that the fucosylated structures of NRG1 had a remarkable increase in BRAF V600E mutant PTC patients compared with BRAF wild type PTC controls.

Conclusions: This study sheds a new light on the role of NRG1 glycosylation in PTC. NRG1 could serve as a supplementary glycobiomarker for BRAF indicator in discrimination of PTC patients with BRAF wild type negative fine needle aspiration results.
\end{abstract}

Keywords: NRG1; lectin; fucosylation; papillary thyroid cancer (PTC)

Submitted Feb 28, 2020. Accepted for publication Jun 16, 2021.

doi: $10.21037 /$ tcr-20-1256

View this article at: https://dx.doi.org/10.21037/tcr-20-1256

\section{Introduction}

Protein glycosylation is an important modification of post-translation playing a crucial role in disease development and progression (1). It has been confirmed that glycosylation changes of serum proteins could affect tumor progression. Protein glycosylation patterns may have totally different effects in human cancer. For example,
$\mathrm{N}$-acetylglucosaminyltransferase $\mathrm{V}$ can catalyze $\beta 6 \mathrm{GlcNAc}$ side chain branching, promoting tumor metastases while $\mathrm{N}$-acetylglucosaminyltransferase III has the opposite effect of inhibiting metastases by synthesizing $\beta 4 \mathrm{GlcNAc}$ (bisecting GlcNAc) (2). Thus, glycosylation changes could be potential biomarkers for cancers and has been a major focus of studies.

Protein glycosylation participates in many biological 
processes, like immune response, ligand- receptor binding and disease (2). Some tumor biological features like tumor metastasis and cell survival rely on the specific cellular glycoforms $(3,4)$. Lectin microarray is a rapid and sensitive high-throughput technique to get glycoconjugate profiles, allowing researchers to directly analyze glycoconjugates like glycoproteins without liberation of glycans from the core substrate (5).

NRG1, also known as neuregulin 1 protein, is found to express in epithelia, glial, neuronal, and skeletal muscle cells. It has been reported that NRG1 overexpression was related in various cancers $(6,7)$. NRG1 protein can bind to ERBB3 or ERBB4 protein, activating ERBB receptor tyrosine kinases, followed by the initiation of signal cascades, including PI3K/AKT pathways (8). NRG1 can be secreted to serum as described (9). Therefore, the serum NRG1 level may play a role in tumorigenesis. Our study is to explore the role of NRG1 in papillary thyroid cancer. However, the glycosylation status of NRG1 and its potential biological function in papillary thyroid cancer remain poorly characterized.

In our study, we used antibody overlay lectin microarray to identify and compare glycosylation status of serum NRG1 in BRAF V600E mutant (BRAF+ PTC patients and BRAF wild type (BRAF-) PTC controls. Specific antibody to NRG1 was utilized to obtain specific signals corresponding to NRG1 glycans in form of lectin affinity profiles with the help of a reproducible, rapid and highthrough put manner. Then we used lectin blot to clarify distinct glycan alterations of serum NRG1 in PTC patients and healthy controls. Finally, serum NRG1 protein level was measured by lectin-ELISA assay following ELISA Index.

We present the following article in accordance with the STARD reporting checklist (available at https://dx.doi. org/10.21037/tcr-20-1256).

\section{Methods}

\section{Serum specimens and clinicopathological data}

The preoperative serum specimens were obtained from patients who underwent thyroidectomy and were diagnosed with papillary thyroid cancer by pathology in Fudan University of Shanghai Cancer Center (FUSCC) from 2018 to 2019. Serum specimens were collected from coagulated blood after centrifugation at a speed of $3,500 \mathrm{r} / \mathrm{min}$ for 5 minutes and then stored at $-80^{\circ} \mathrm{C}$ until use. The study was conducted in accordance with the Declaration of Helsinki (as revised in 2013). This study was approved by the Research Ethics Committee of FUSCC and the Institutional Review Board of the National Cancer Center (approval number: 050432-4-1911D). Each patient provided a signed informed consent for his/her specimens and information to be used for research.

\section{Immunobistochemical (IHC) staining of BRAF V600E}

IHC staining was carried out according to the manufacture's protocol. Firstly, formalin-fixed and paraffin-embedded tissue sections were deparaffinized in xylene and hydrated by descending concentrations of ethanol before being placed in a blocking solution so that the endogenous peroxidase activity was inhibited. Then we incubated the slides using primary antibodies (rabbit anti-human BRAF V600E, 1:400 dilution, Abcam, USA) at $4{ }^{\circ} \mathrm{C}$ overnight. Next, a horseradish peroxidase-conjugated rabbit secondary antibody was added for 1 hour at room temperature, followed by 3,3'-diaminobenzidine (DAB) development (DAB Substrate Chromogen System, Dako Agilent Technologies, Shanghai, China) and hematoxylin and eosin (H\&E) as per standard staining protocol. Slides were fixed and pictures were obtained with the Olympus IX71 inverted microscope using the DP2-BSW Olympus image acquisition software system. Finally, three experienced pathologists who were blinded to the clinicopathologic data confirmed the results.

\section{Immunoprecipitation and antibody overlay lectin microarray}

We used Pierce Direct IP Kit (Thermo, Rockford, USA) and monoclonal NRG1 antibody (Santa Cruz, Texas, USA) (sc-393006, E-12) according to the manufacture's protocol for NRG1 protein immunoprecipitation from pooling sera of ten $\mathrm{BRAF}(+)$ PTC patients and ten $\mathrm{BRAF}(-)$ PTC controls, respectively. An equal amount of purified NRG1 protein were diluted with $100 \mu \mathrm{L}$ PBS containing $1 \%$ Triton X-100 (PBSTx) and applied to the lectin microarray including 50 lectins in total. Each lectin had 6 repeated spots. After incubation at $20^{\circ} \mathrm{C}$ for 12 hours, the lectin microarray was added with $20 \mu \mathrm{g}$ non-labeled human polyclonal IgG to block glycoprotein at room temperature for 30 minutes. Then we used PBSTx to rinse the glass slide for three times. We added $100 \mu \mathrm{L}$ biotinylated NRG1 antibody solution into the microarray with another 
incubation at room temperature for one hour. After three times of washing with PBSTx, we added $60 \mu \mathrm{L}$ Cy5-labeled streptavidin (Invitrogen, MD, USA) solution to the lectin microarray and incubated it at room temperature for half an hour. Finally, we washed the lectin microarray again using TBSTx and tested with an evanescent-field fluorescence scanner (Capitalbio, Beijing, China). Array Pro Analyzer, version 4.5 (Media Cybernetics) software was used to obtain the signal intensity of both spot and background. When their ratio was over 1.5 , the spot intensity should be considered valid. The intensity of each spot was defined as the signal intensity subtracting the background intensity. The average intensity of 6 repeated spots represented the corresponding lectin intensity value (10).

\section{Lectin blot assay}

Purified serum NRG1 from ten $\operatorname{BRAF}(+)$ PTC patients and ten BRAF(-) PTC controls were separated by $10 \%$ sodium dodecyl sulfate-polyacrylamide gel electrophoresis (SDSPAGE). Proteins were then transferred to polyvinylidene fluoride membrane (Millipore, Billerica, USA) according to conventional protocol. After being blocked by $3 \%$ bull serum albumin (BSA) in TBS containing 1\% Tween 20 (TBST) at room temperature for one hour, the membrane was then incubated with biotinylated lectin at room temperature overnight. Next, we washed the membrane with TBST for three times, followed by the incubation with avidin-HRP at room temperature for another 1 hour. Then we washed the membrane with TBST for another three times. Meanwhile, western blotting was also applied for the same purified protein of NRG1. After being blocked with $5 \%$ nonfat milk in TBST at room temperature for one hour, the membrane containing transferred proteins was then incubated with NRG1 antibody (Santa Cruz, Texas, USA) overnight and finally washed with TBST for 3 times. Following incubation in a solution of goat anti-rabbit (1:1,000, Santa Cruz, USA) or anti-mouse IgG (1:5,000 for both; Santa Cruz, USA) at room temperature for 1 hour, we could visualize both the immunoreactive protein bands and the lectin blot bands at the same time with the assistance of the enhanced chemiluminescence (ECL) detection system (GE Healthcare, Piscataway, NJ, USA) (10).

\section{Enzyme-linked immunosorbent assay (ELISA) of NRG1}

The ELISA kits for NRG1 protein were obtained from Guangzhou Institute of Biomedicine and Health, Chinese
Academy of Sciences. Two 96-well plates were used for Aleuria aurantia lectin (AAL) ELISA and Protein ELISA, respectively. Plate1 for AAL ELISA was blocked by 3\% BSA in PBS ( $\mathrm{pH} 7.2-7.4)$ at room temperature for one hour. Oxidation buffer $\left(100 \mathrm{mM} \mathrm{NaIO}_{4}, 50 \mathrm{mM}\right.$ citric acid, $\mathrm{pH}$ 4.0) was prepared to react antibodies which were coated on the plate at $4^{\circ} \mathrm{C}$ for 1 hour. Then $100 \mu \mathrm{L}$ nondiluted serum was added to each well and incubated for two hours. After 4 times of washing with PBST (PBS with $0.05 \%$ Tween $20, \mathrm{pH} 7.2-7.4), 1 \mu \mathrm{g} / \mathrm{mL}$ biotinylated AAL (Vector, Burlingame, CA, USA) was applied at room temperature for 2 hours. Finally, HRP-streptavidin was applied to each well, followed by TMB working solution and stop solution in order. The OD value at $450 \mathrm{~nm}$ of fucosylated NRG1 protein was obtained from NanoQuant Infinite M200 (TECAN, Switzerland). The blocking buffer (1\% BSA, $0.05 \% \mathrm{NaN}_{3}$ with PBS, $\mathrm{pH}$ 7.2-7.4) was applied to plate 2 for protein ELISA at room temperature for one hour. $100 \mu \mathrm{L}$ of 1.4-fold diluted serum sample with PBS was added to each well of plate 2. After two hours of incubation, it was washed by PBST for 4 times, followed by detection antibody for another 2 hours incubation at room temperature. Finally, TMB working solution was applied to the well followed by the stop solution. OD value at $450 \mathrm{~nm}$ of NRG1 was measured in the same way as plate 1 . There were $60 \mathrm{BRAF}(-)$ PTC patients and $60 \mathrm{BRAF}(+)$ PTC patients who were tested individually by NRG1 protein ELISA and AAL ELISA at the same time (11).

\section{Statistical analysis}

All statistical analyses were conducted by SPSS 22.0 (SPSS, Chicago, IL, USA) for Windows. Data were shown as the mean \pm SD unless otherwise indicated. We assessed the normality distribution of data before using Student's test (two-tailed) to compare two groups of continuous variables. Chi-square and Fisher's exact tests were used for categorical variables. $\mathrm{P}<0.05$ was considered to be statistically significant.

\section{Results}

\section{Antibody overlay lectin microarray and lectin blot showed glycan alternations of serum NRG1}

The clinicopathological data of the patients were abstracted and shown in Table 1. An antibody overlay microarray including fifty different lectins was used to analyze the 
different glycan alterations of NRG1 from sera of $\mathrm{BRAF}(+)$ PTC and BRAF(-) PTC patients. Equal amount of immunoprecipitated NRG1 protein was applied to the microarray and the result was shown in Figure 1. We got fourteen lectins with their affinity glycan profiles in total, including GNL, GSL2, AAL, BPL, ECL, CAL, NML, HHL, PHA-L, RCA-I, ConA, DBA, PWA and LEL (Figure 2A). Six of fourteen lectins, namely, GSL2, BPL, NML, HHL, PHA-L and LEL had significantly increased binding affinity capacity in $\mathrm{BRAF}(+)$ PTC compared with $\mathrm{BRAF}(-)$ PTC controls $(\mathrm{P}<0.05$, Figure $2 B)$.

Next, we conducted lectin blot assay of human serum NRG1 to determine the signal pattern that how lectin

\begin{tabular}{lcc}
\multicolumn{3}{l}{ Table 1 Characteristics of $\mathrm{PTC}^{\mathrm{BRAF}+}$ and $\mathrm{PTC}^{\mathrm{BRAF}-}$ patients } \\
\hline Group & $\mathrm{PTC}^{\mathrm{BRAF}+}$ & $\mathrm{PTC}^{\mathrm{BRAF}-}$ \\
\hline Number & 60 & 60 \\
Sex (F/M) & $42 / 18$ & $39 / 21$ \\
Age (years) & $45.7 \pm 10.6$ & $56.2 \pm 10.8$ \\
Tumor size (cm) & $1.4 \pm 0.6$ & $1.3 \pm 0.3$ \\
Multifocal lesions & 23 & 34 \\
Bilateral & 35 & 24 \\
Lymph node metastasis & 18 & 12 \\
TNM staging (I-II/III-IV) & $51 / 9$ & $58 / 2$ \\
\hline
\end{tabular}

Papillary thyroid cancer diagnosis was confirmed by ultrasound imaging and biopsy. BRAF mutations were detected by immunohistochemical BRAF V600E (VE1) antibodies and confirmed by three pathologists. microarray correlated with $\mathrm{BRAF}(+)$ and $\mathrm{BRAF}(-)$ PTC patients. For better understanding of proteoglycan levels, we defined the lectin/NRG1 ratio as the intensity of the band in lectin blot, divided by the intensity of the band in Western blot. Figure 3 showed that LEL, BPL and NML tended to have bound to NRG1 in BRAF(+) PTC patients compared to $\mathrm{BRAF}(-)$ controls. All the three lectins were found to bind to $\alpha$-linked fucose related structures.

\section{AAL ELISA and protein ELISA measured Fuc-NRG1 level in PTC with BRAF(+) and BRAF(-) patients}

For preclinical assessment of fucosylated NRG1, we selected AAL as the targeted lectin because of its well performance in lectin-ELISA assay as reported. In ALL ELISA, NaIO4 was used to oxidize the glycan of the antibody to block the binding of AAL to the antibodies. Protein ELISA was performed according to standard procedures. Standard curves were built before sera samples measurement to determine the detective limitation in ALL ELISA and protein ELISA. Considering the low concentration of NRG1 in sera samples, we used 1.4-fold diluted serum for protein ELISA and undiluted serum for AAL ELISA. In order to ensure the accuracy of AAL ELISA, three experiments were performed independently using different mixed serum, and each experiment was measured three times. We calculated the average coefficient of variance to be $4.7 \%$, indicating that the AAL ELISA results were reproducible (Table 2).

The ELISA index was used to measure sixty $\operatorname{BRAF}(+)$ PTC serum samples and 60 serum samples from BRAF(-)
A

\begin{tabular}{|l|l|l|l|}
\hline 1 & Positive control & Blank & WGA \\
\hline 2 & PWA & PCL & VAL \\
\hline 3 & UEA & STL & NML \\
\hline 4 & BPL & SBA & CAL \\
\hline 5 & RCA II & VVL & PHA-L \\
\hline 6 & PTL I & PSA & EEL \\
\hline 7 & PHA-E & SJA & DBA \\
\hline 8 & PAL & PNA & NPL \\
\hline 9 & RCA I & PTLII & MAL I \\
\hline 10 & WFL & LTL & LPL \\
\hline 11 & GSLI & LCA & JAC \\
\hline 12 & HPL & GLS2 & HAL \\
\hline 13 & ACL & MPL & ECL \\
\hline 14 & GNL & HHL & GNL \\
\hline 15 & AAL & MAL I & DSA \\
\hline 16 & ConA & CFL & RIC \\
\hline 17 & LEL & ABL & GSL I b4 \\
\hline 18 & CSL & blank & Positive control \\
\hline \multicolumn{4}{|l}{} \\
\hline
\end{tabular}

B

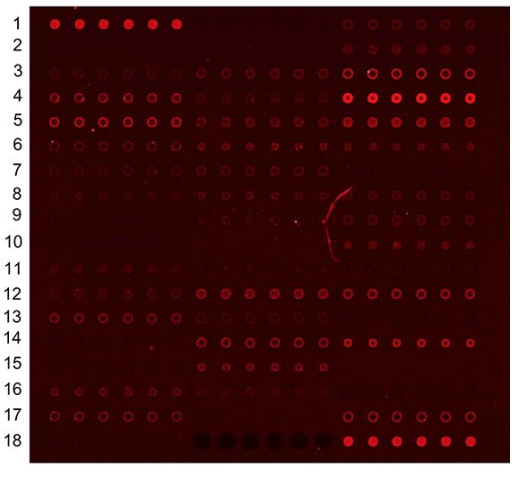

$\operatorname{BRAF}(+)$

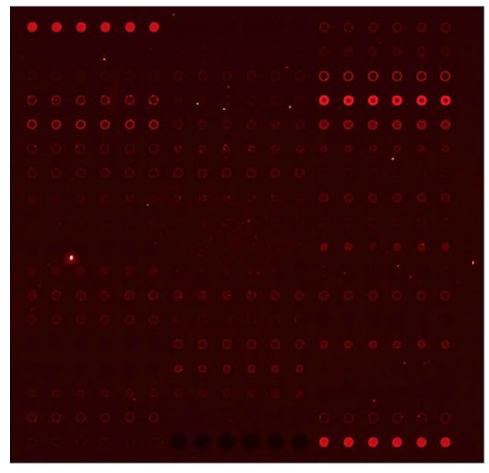

$\operatorname{BRAF}(-)$

Figure 1 Detection of the glycan pattern of NRG1 by antibody overlay lectin microarray. (A) The lectin microarray format containing fifty different binding lectin spots. (B) Typical glycan profiles of NRG1 of patients with BRAF(+) and BRAF(-) papillary thyroid cancer. 
A

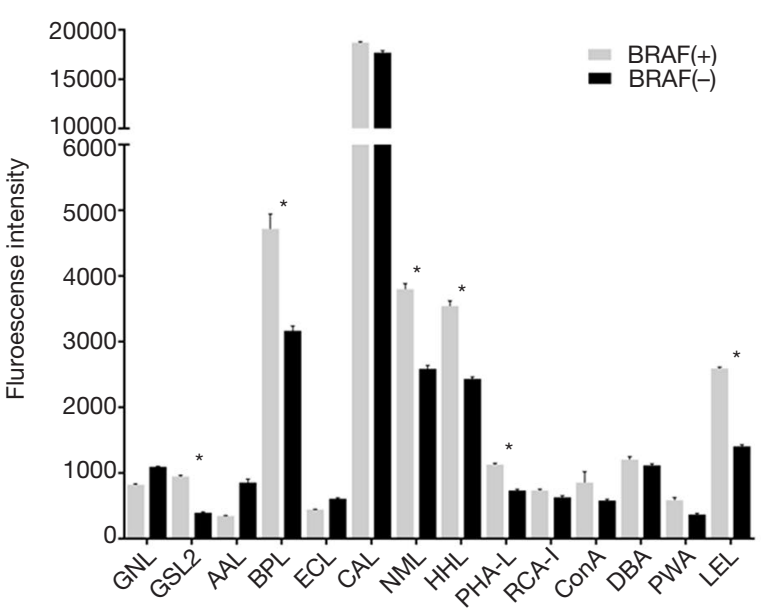

B

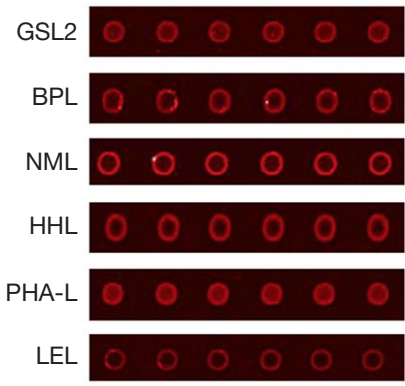

$\operatorname{BRAF}(+)$

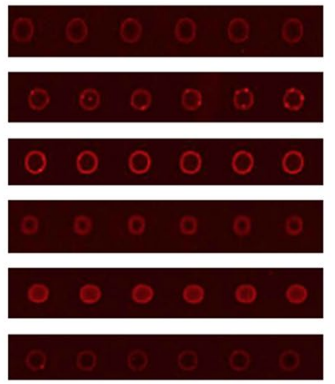

$\operatorname{BRAF}(-)$

Figure 2 Significant lectins binding profiles of NRG1. (A) The signals of fourteen lectins were detected in the NRG1 glycans of patients with $\mathrm{BRAF}(+)$ and BRAF(-) PTC. The differences $(\mathrm{P}<0.05)$ in the signal patterns of the six lectins between the two groups were labeled $(*)$. (B) The magnification spots of the six statistically different lectins (GSL2, BPL, NML, HHL, PHA-L and LEL) in BRAF(+) and BRAF(-) papillary thyroid cancer patients.

A

NRG1

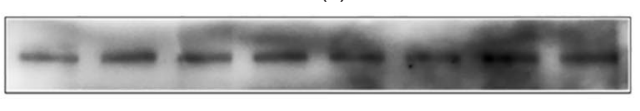

BPL

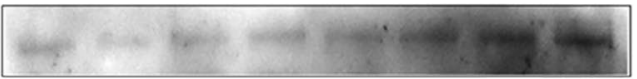

NML

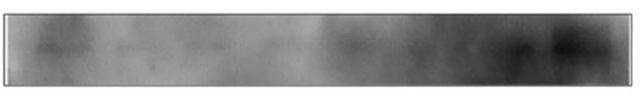

LEL

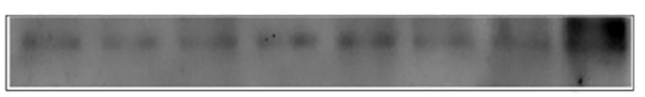

$\operatorname{BRAF}(-)$

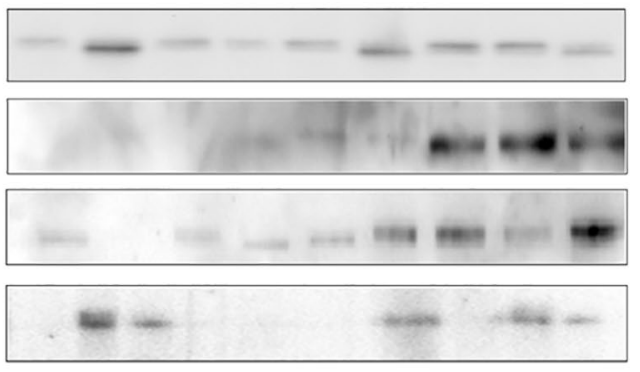

B
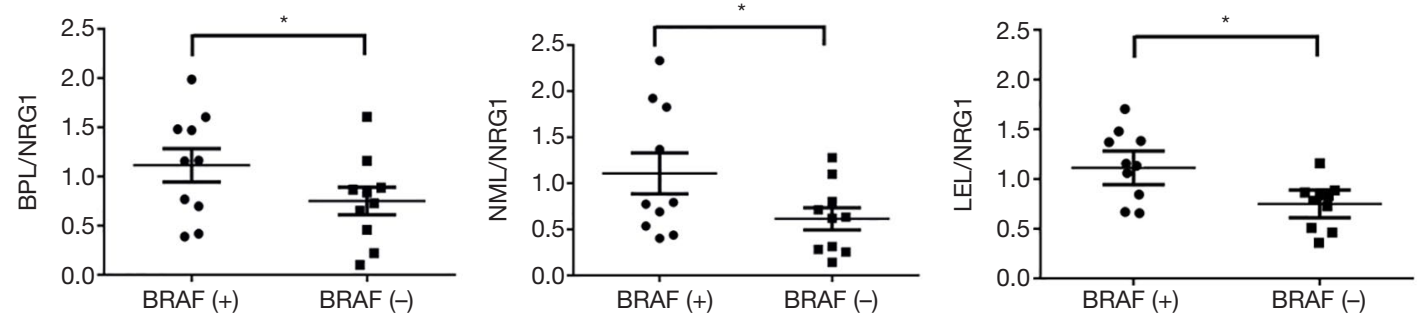

Figure 3 Confirmation of glycan changes in purified NRG1 in patients with BRAF(+) and BRAF(-) PTC. (A) Lectin blot and western blot analyzed the immunoprecipitated NRG1 of $10 \mathrm{BRAF}(+)$ and $10 \mathrm{BRAF}(-)$ PTC patients. (B) Scatter plot of lectin/NRG1 ratio (intensity of band from lectin blot/ intensity of band from western blot). *, $\mathrm{P}<0.05$.

PTC patients. The ELISA index was calculated as the ratio of the OD value of protein NRG1 to fucosylated NRG1. Table 3 showed that the ELISA index of Fuc-NRG1 of $\mathrm{BRAF}(+)$ patients was significantly higher than that of BRAF(-) patients.

\section{Discussion}

NRG1 is a protein located on cell membrane, whose isoforms can be produced from the NRG-1 gene by alternative splicing. Its biology is complicated by the fact that multiple splice variants are produced from the NRG- 
Table 2 Three independent measurements of AAL ELISA using different pooled sera sample

\begin{tabular}{lcc}
\hline Pooled sera sample $(\mathrm{n}=3)^{\mathrm{a}}$ & $\mathrm{OD}_{450}^{\mathrm{b}}$ & $\mathrm{CV} \%$ \\
\hline 1 & $0.62732 \pm 0.0554$ & 9.70 \\
2 & $1.2106 \pm 0.0161$ & 1.80 \\
3 & $0.7322 \pm 0.0266$ & 2.71 \\
\hline
\end{tabular}

${ }^{\mathrm{a}}$ Each pooled sera sample was mixed with three different sera. ${ }^{b}$ Each pooled sera was measured three times. CV, coefficient of variance.

Table 3 Comparison of Fuc-NRG1 in $60 \mathrm{PTC}^{\mathrm{BRAF}+}$ and 60 PTC BRAF- patients

\begin{tabular}{lcc}
\hline Group & Cases & Fuc-NRG1 (mean \pm SD) \\
\hline PTC $^{\text {BRAF+ }}$ & 60 & $7.64 \pm 6.14$ \\
PTC $^{\text {BRAF- }}$ & 60 & $2.85 \pm 1.50$ \\
$t$-test & & $\mathrm{P}<0.0001$ \\
\hline
\end{tabular}

1 gene (12). NRG1 is reported to be upregulated in many various cancers. However, there are few researches on its glycosylation alterations related with BRAF mutant status in PTC patients (13). Therefore, we focused on serum NRG1 protein level of PTC patients and analyzed the relevance between NRG1 glycosylation and PTC clinical features. Changes in glycosylation of serum glycoproteins are related to tumor development. Our study used antibody overlay lectin microarray for assessing glycosylation status of NRG1 in BRAF(+) PTC and BRAF(-) PTC patients. Six reactive fractions of NRG1, including GSL2, BPL, NML, HHL, PHA-L and LEL, were found to be increased in $\mathrm{BRAF}(+)$ PTC patients. This result was validated by lectin blot and the change in fucosylation structure were proved to be significant.

BRAF V600E gene mutation has been widely proven to be associated with lymph node metastasis, extrathyroid extension, recurrence and mortality and is considered a potential target for treatment of papillary thyroid cancer (14). Therefore, it is of great importance to identify BRAF V600E status. Clinicians infer benign and malignant by measuring BRAF levels in thyroid fine-needle biopsy tissue. Our study recruited BRAF(-) PTC patients instead of healthy people as a control group to discover of differential glycobiomarkers related with BRAF in PTC. BRAF(+) PTC patients had significantly higher fucosylation compared with BRAF(-) PTC patients. Therefore, we speculated that higher fucosylation of serum NRG1 may be related with BRAF V600E mutation status. The results indicated that NRG1 may have biological function in thyroid disease progression, especially in PTC (15). The glycobiology functions of NRG1 in thyroid are still poorly known and deep research is needed.

As a high-throughput system, the antibody overlay lectin microarray is applied for analyzing glycan patterns of specific glycoproteins (16). With the help of this system, we successfully identified fourteen reactive fractions of NRG1 in $\mathrm{BRAF}(+)$ PTC and BRAF(-) PTC patients, including GNL, GSL2, AAL, BPL, ECL, CAL, NML, HHL, PHA-L, RCA-I, ConA, DBA, PWA and LEL. Therefore, we can combine the ELISA assay with lectin detection and apply it to clinical glycobiomarkers on a large scale. The main limitation of our study was the small sample size and the retrospective nature of the data analysis which could led to a selection bias.

In conclusion, we used multiple lectin assays and successfully confirmed the significantly elevated Fuc-NRG1 in BRAF(+) PTC patients compared with BRAF(-) PTC patients. They are practical tools for detecting changes in glycosylation of target glycoproteins, providing us a potential role of NRG1 glycosylation in PTC.

\section{Acknowledgments}

Thanks to Jiang Kai for his selfless help and guidance in the experiment.

Funding: This work was supported by funds from the National Natural Science Foundation of China (National Natural Science Foundation of China, Grant No: 81502317 to $\mathrm{WJW}$ ).

\section{Footnote}

Reporting Checklist: The authors have completed the STARD reporting checklist. Available at https://dx.doi. org/10.21037/tcr-20-1256

Data Sharing Statement: Available at https://dx.doi. org/10.21037/tcr-20-1256

Peer Review File: Available at https://dx.doi.org/10.21037/ tcr-20-1256

Conflicts of Interest: All authors have completed the ICMJE uniform disclosure form (available at https://dx.doi. org/10.21037/tcr-20-1256). The authors have no conflicts 
of interest to declare.

Ethical Statement: The authors are accountable for all aspects of the work in ensuring that questions related to the accuracy or integrity of any part of the work are appropriately investigated and resolved. The study was conducted in accordance with the Declaration of Helsinki (as revised in 2013). This study was approved by the Research Ethics Committee of FUSCC and the Institutional Review Board of the National Cancer Center (approval number: 050432-4-1911D). Each patient provided a signed informed consent for his/her specimens and information to be used for research.

Open Access Statement: This is an Open Access article distributed in accordance with the Creative Commons Attribution-NonCommercial-NoDerivs 4.0 International License (CC BY-NC-ND 4.0), which permits the noncommercial replication and distribution of the article with the strict proviso that no changes or edits are made and the original work is properly cited (including links to both the formal publication through the relevant DOI and the license). See: https://creativecommons.org/licenses/by-nc-nd/4.0/.

\section{References}

1. Oliveira-Ferrer L, Legler K, Milde-Langosch K. Role of protein glycosylation in cancer metastasis. Semin Cancer Biol 2017;44:141-52.

2. Ramakrishnan B, Boeggeman E, Qasba PK. Binding of N-acetylglucosamine (GlcNAc) $\beta 1$-6-branched oligosaccharide acceptors to $\beta 4$-galactosyltransferase I reveals a new ligand binding mode. J Biol Chem 2012;287:28666-74.

3. Moremen KW, Tiemeyer M, Nairn AV. Vertebrate protein glycosylation: diversity, synthesis and function. Nat Rev Mol Cell Biol 2012;13:448-62.

4. Hart GW, Slawson C, Ramirez-Correa G, et al. Cross talk between O-GlcNAcylation and phosphorylation: roles in signaling, transcription, and chronic disease. Annu Rev Biochem 2011;80:825-58.

Cite this article as: Liu WL, Cao YM, Liao T, Qu N, Zhu YX, Wei WJ. Multiple lectin assays in detecting glycol-alteration status of serum NRG1 in papillary thyroid cancer. Transl Cancer Res 2021;10(7):3218-3224. doi: 10.21037/tcr-20-1256
5. Hirabayashi J, Yamada M, Kuno A, et al. Lectin microarrays: concept, principle and applications. Chem Soc Rev 2013;42:4443-58.

6. Lin CW, Mostafa NM, L Andress D, et al. Relationship Between Atrasentan Concentrations and Urinary Albumin to Creatinine Ratio in Western and Japanese Patients With Diabetic Nephropathy. Clin Ther 2018;40:242-51.

7. Heining C, Horak P, Uhrig S, et al. NRG1 Fusions in KRAS Wild-Type Pancreatic Cancer. Cancer Discov 2018;8:1087-95.

8. Sudol M. Neuregulin 1-activated ERBB4 as a "dedicated" receptor for the Hippo-YAP pathway. Sci Signal 2014;7:pe29.

9. Vasti C, Hertig CM. Neuregulin-1/erbB activities with focus on the susceptibility of the heart to anthracyclines. World J Cardiol 2014;6:653-62.

10. Jiang K, Shang S, Li W, et al. Multiple lectin assays for detecting glyco-alteration of serum GP73 in liver diseases. Glycoconj J 2015;32:657-64.

11. Shimomura M, Nakayama K, Azuma K, et al. Establishment of a novel lectin-antibody ELISA system to determine core-fucosylated haptoglobin. Clin Chim Acta 2015;446:30-6.

12. Lemmens K, Doggen K, De Keulenaer GW. Role of neuregulin-1/ErbB signaling in cardiovascular physiology and disease: implications for therapy of heart failure. Circulation 2007;116:954-60.

13. He H, Li W, Liyanarachchi S, et al. The Role of NRG1 in the Predisposition to Papillary Thyroid Carcinoma. J Clin Endocrinol Metab 2018;103:1369-79.

14. Xing M, Alzahrani AS, Carson KA, et al. Association between BRAF V600E mutation and recurrence of papillary thyroid cancer. J Clin Oncol 2015;33:42-50.

15. Son HY, Hwangbo Y, Yoo SK, et al. Genome-wide association and expression quantitative trait loci studies identify multiple susceptibility loci for thyroid cancer. Nat Commun 2017;8:15966.

16. Ribeiro JP, Mahal LK. Dot by dot: analyzing the glycome using lectin microarrays. Curr Opin Chem Biol 2013;17:827-31. 